\title{
Primary hepatocellular carcinoma in a patient with history of treated breast cancer: a case report with challenging diagnosis and treatment
}

This article was published in the following Dove Press journal: International Journal of General Medicine

\author{
Christoforos Kosmidis' \\ Nikolaos Varsamis' \\ Georgios Anthimidis' \\ Sofia Baka ${ }^{2}$ \\ Dimitrios Valoukas ${ }^{3}$ \\ Triantafyllia Koletsa ${ }^{4}$ \\ Katerina Zarampouka ${ }^{4}$ \\ Georgios Koimtzis ${ }^{5}$ \\ Eleni Georgakoudi ${ }^{6}$ \\ Paul Zarogoulidis ${ }^{7}$ \\ Christoforos Efthymiadis' \\ 'Department of Surgery, Interbalkan \\ European Medical Center, \\ Thessaloniki, Greece; ${ }^{2}$ Department \\ of Oncology, Interbalkan European \\ Medical Center, Thessaloniki, \\ Greece; ${ }^{3}$ Department of Oncology, \\ General Hospital of Ptolemaida, \\ Ptolemaida, Greece; ${ }^{4}$ Pathology \\ Department, Faculty of Medicine, \\ Aristotle University of Thessaloniki, \\ Thessaloniki, Greece; ${ }^{5}$ Third \\ Surgical Department, Faculty of \\ Medicine, Aristotle University of \\ Thessaloniki, Thessaloniki, Greece; \\ ${ }^{6}$ Aristotle University of Thessaloniki, \\ Thessaloniki, Greece; "Pulmonary- \\ Oncology Unit, "Theageneio" Cancer \\ Hospital, Thessaloniki, Greece
}

Correspondence: Paul Zarogoulidis

Pulmonary-Oncology Unit, "Theageneio"

Cancer Hospital, 64 Panorama Street, PO

Box 55326, Thessaloniki, Greece

Tel +306977271974

Email pzarog@hotmail.com
Introduction: Breast cancer is the most common malignancy in women worldwide. Longterm survivors among patients treated for breast cancer are at a high risk for developing a second primary malignancy. Hepatocellular carcinoma is the most frequent primary hepatic malignancy and should be ruled out in breast cancer patients who are diagnosed with solitary hepatic lesions. False diagnosis may lead to inappropriate oncologic staging and treatment of the disease.

Case presentation: We present the case of a 73-year-old female patient who had been treated for invasive ductal breast cancer 7 years ago and was diagnosed with a solid hepatic lesion at segments VI and VII and a small, calcified lesion at the tail of the pancreas on follow-up with an abdominal computed tomography. Oncology council decided that both lesions could be resected after determining whether they were metastatic or second primary malignancies. The patient underwent laparotomy and rapid biopsy which showed primary hepatocellular carcinoma and fibrosis of the pancreas. We performed hepatic segmentectomy (VI-VII) and cholecystectomy, while the pancreatic lesion was left intact. The postoperative course of the patient was uncomplicated and she remains disease free 2 years after the operation without any adjuvant therapy. Conclusion: All hepatic lesions detected in breast cancer patients should be evaluated with open mind and liver biopsy should be performed to get a definitive diagnosis and implement the proper treatment strategy.

Keywords: breast cancer, hepatocellular cancer, second primary malignancy, rapid biopsy, microwave tissue coagulator

\section{Introduction}

Breast cancer is the most frequently diagnosed malignancy among women worldwide. Multidisciplinary treatment has improved the overall survival rate among early-stage patients, which is reported to be $88.8 \%$ at 5 years for Caucasian females. An increasing number of patients treated for breast cancer are at risk of developing a second primary malignancy during medical follow-up. ${ }^{1-3}$ Metastatic disease rate is $\sim 30 \%$ and represents the leading cause of deaths in breast cancer patients. Solitary breast cancer hepatic metastases occur in 5\%-25\% of cases, and differential diagnosis should include primary hepatocellular carcinoma (HCC) especially at high-risk patients. False diagnosis may lead to inappropriate oncologic staging and treatment of the disease. ${ }^{1,4-6}$ We present the case of a patient who had been treated for invasive ductal breast cancer 7 years ago and was found to have both a solitary hepatic and a pancreatic lesion. 


\section{Case presentation}

Our patient is a 73-year-old Caucasian female with medical history of hypertension and type 2 diabetes. She had been treated for invasive ductal breast cancer 7 years ago with lumpectomy of the left breast and axillary lymph node dissection. Radiation therapy and seven cycles of adjuvant chemotherapy were administered to her postoperatively, and Anastrozole was prescribed to her for 5 years. She underwent regular annual follow-up for 5 years with chest and abdominal computed tomography (CT) scanning with no signs of recurrence or metastatic disease. Seven years after the initial treatment for breast cancer, abdominal CT revealed a solid hepatic lesion at segments VI and VII measuring $7 \mathrm{~cm}$ in diameter as well as a $1 \mathrm{~cm}$ calcified lesion at the tail of the pancreas (Figure 1, left). Tumor markers AFP, CA 15-3, CA 19-9, and CEA were all within normal limits and the patient did not have any symptoms or signs of abdominal mass apart from mild tenderness on palpation of the right subcostal region. Moreover, biochemical markers of liver and pancreatic function were normal.

Subsequently the patient went through oncologic consultation, and both lesions were considered to be resectable, although controversy arose whether they were metastatic or second primary malignancies. CT-guided percutaneous biopsies or laparoscopic biopsies were proposed as minimal invasive methods of diagnosis. The patient rejected both of them due to the risk for possible complications and cancer spillage and decided to undergo laparotomy, open rapid biopsy, and excision of the lesions in one procedure. After accessing the peritoneal cavity through an extended right subcostal incision, we performed hepatic mobilization and exposure of the right hepatic lobe. A solid mass was recognized at hepatic segments VI and VII and rapid biopsy was taken which showed primary HCC. Moreover, after entering the lesser sac, a second rapid biopsy was taken from the lesion at the pancreatic tail which showed chronic fibrosis without elements of malignancy (Figure 2A). We decided to perform resection of hepatic segments VI and VII since intraoperative hepatic ultrasound also confirmed that the mass was resectable with safe oncologic margins. Hepatic segmentectomy was achieved using a microwave tissue coagulator to mark and coagulate the margins of hepatectomy and ultrasound harmonic scalpel to transect the hepatic parenchyma with a minimal blood loss of $40 \mathrm{cc}$ (Figure 3). Moreover, argon beam coagulator and fibrin glue were used to seal the raw liver surface and prophylactic cholecystectomy was also performed. Finally, the pancreatic lesion was left intact since malignancy was not confirmed with rapid biopsy.

Pathology report described a hepatic mass with maximum diameter of $6.5 \mathrm{~cm}$ which consisted of malignant cells with mild to severe atypia and low to medium mitotic rate. Immunohistochemistry showed that cancer cells highly expressed keratins 8/18, Hep-Par 1 and AFP and did not express keratin 7 and GATA-3 (Figure 2B-D). Endothelial cells within the mass were positive for $\mathrm{CD} 34$. The gallbladder along with the cystic lymph node was normal. The final diagnosis was low-grade HCC with liver margins widely free from malignant infiltration. ${ }^{7-9}$

The postoperative course of the patient was uneventful. The subdiaphragmatic and subhepatic drains were removed

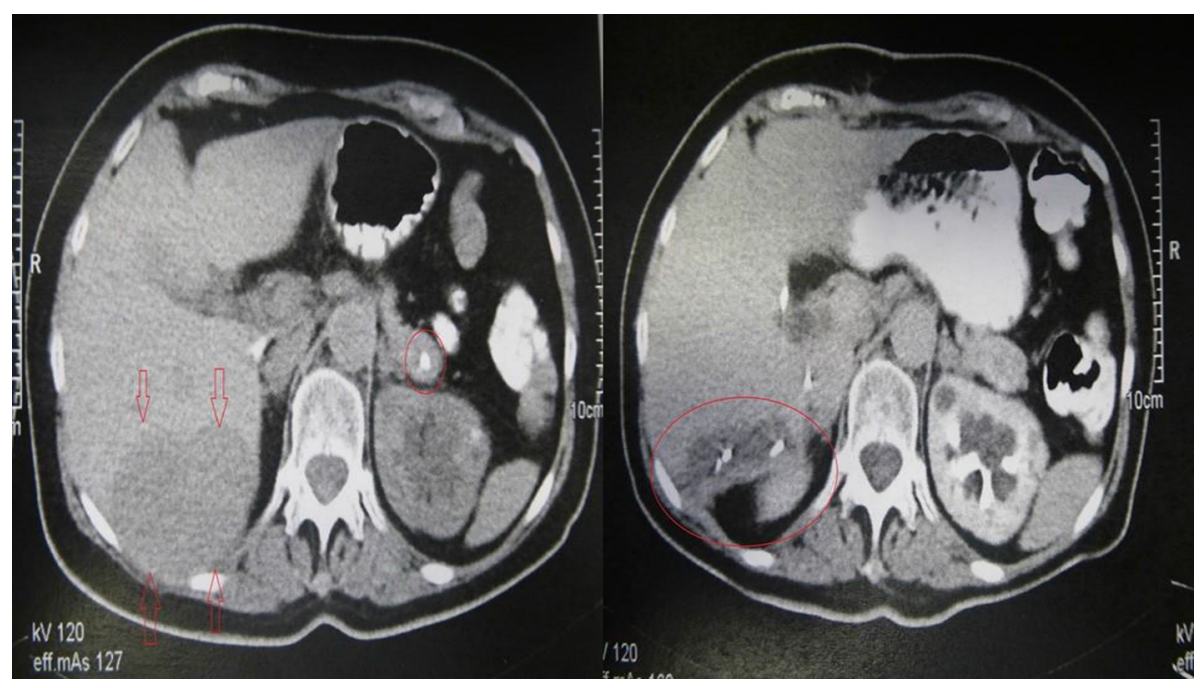

Figure I Left: Preoperative abdominal CT. Solid hepatic lesion at segments VI and VII (arrows) and calcified lesion at the tail of the pancreas (circle). Right: Postoperative abdominal CT. Subcapsular hepatic fluid collection without recurrence of the HCC (circle).

Abbreviations: CT, computed tomography; HCC, hepatocellular carcinoma. 


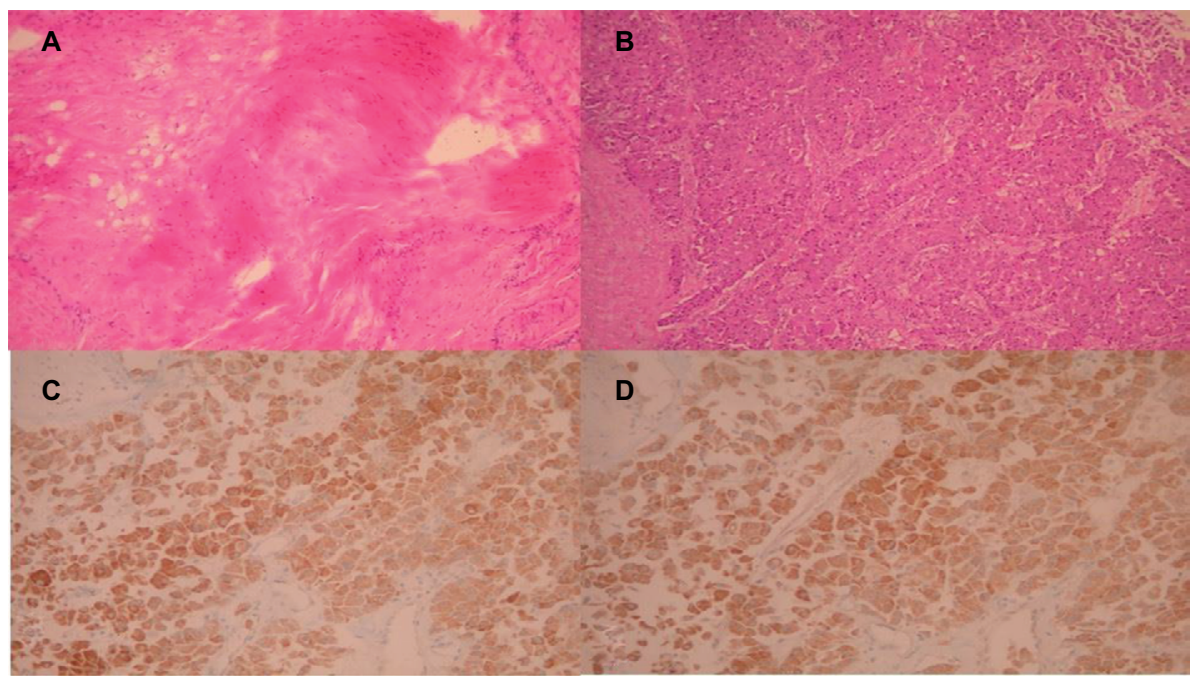

Figure 2 (A) Scar lesion from the pancreas (H\&E I00X). (B) The tumor cells are arranged in abnormal and thick trabeculae (H\&E I00X). (C) The neoplastic cells are positive for HepParl (Immunostain 200X). (D) The neoplastic cells are positive for AFP (Immunostain 200X).

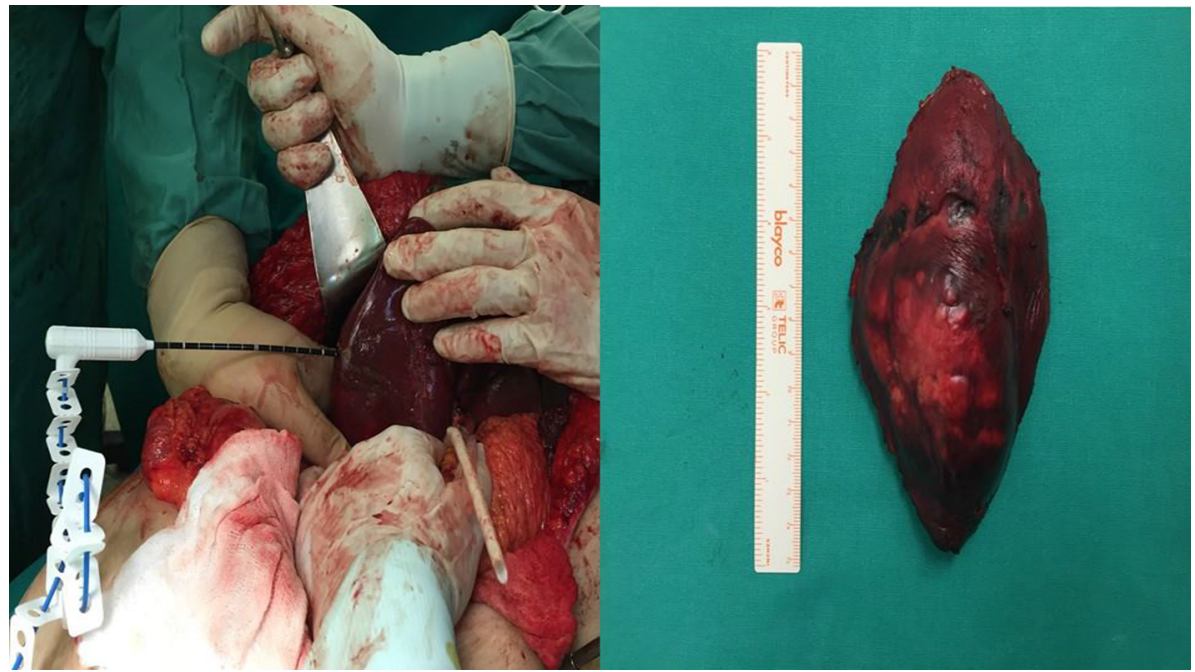

Figure 3 Left: Intraoperative photo. Microwave tissue coagulator marking the margins for hepatic segmentectomy. Right: Gross specimen photo. Hepatic segments VI and VII containing the HCC.

Abbreviation: HCC, hepatocellular carcinoma.

on the third postoperative day without signs of hemorrhage or bile leakage and the patient was discharged on the fifth postoperative day. Follow-up abdominal CT scanning on the first and sixth month after the operation showed subcapsular hepatic fluid collection without recurrence of the HCC (Figure 1, right).The patient remains disease free 2 years after the hepatic segmentectomy without any adjuvant therapy.

Written informed consent obtained from the patient is for publication of the case details and any accompanying images.

\section{Discussion}

Long-term survivors among patients treated for breast cancer are at a higher risk for developing a second primary malignancy according to Warren and Gates criteria compared with the general population. This occurs in about $12 \%$ of the affected women, and they comprise $25 \%$ of the total multiple cancer patients. This risk has mainly been associated with adjuvant therapy. Chemotherapy is linked to acute myeloid leukemia and myelodysplastic syndrome, hormonal therapy with Tamoxifen predisposes to uterine cancer and radiation therapy increases the risk for sarcomas and lung cancer. Finally, the incidence of gastrointestinal malignant tumors has been reported high in breast cancer survivors. ${ }^{1-3,7,8}$

Liver is the third commonest site for breast cancer metastasis after the bony skeleton and the lungs. Median survival in these cases is $\sim 4$ months with very low 5 -year survival. Solitary hepatic breast cancer metastases eligible for surgical 
resection occur in $\sim 5 \%$ of the patients. Multiple studies have confirmed that margin negative hepatectomy in these carefully selected patients although cytoreductive in nature offers a survival advantage over standard chemotherapy regimens and can be done safely. ${ }^{4-6,9-13}$

Metastatic disease of the pancreas from a distant malignancy is very rare, and there are only 12 reported cases originating from breast cancer. Although pancreatic resection has been described in some of these cases, they are associated with poor prognosis. ${ }^{14,15}$

Our patient is a 7-year survivor after multidisciplinary treatment for breast cancer who presented with two suspicious lesions at the liver and the pancreas. The incidence of liver metastasis in breast cancer patients is much higher than that of second primary HCC. HCC is the commonest primary hepatic malignancy and is strongly associated with cirrhosis, chronic hepatitis $\mathrm{B}$ and $\mathrm{C}$ infection and alcoholic liver disease. Previous long-term Tamoxifen therapy has been described in a case of second $\mathrm{HCC}$, but in general $\mathrm{HCC}$ is not associated with adjuvant therapy for breast cancer like other second malignancies mentioned before. Diagnosis may be clinically confirmed in high-risk patients with elevated AFP levels and typical imaging features at four-phase dynamic contrast enhanced CT. ${ }^{16-19}$ Our patient did not belong to a high-risk group and had normal AFP levels, and the hepatic lesion showed only arterial enhancement on CT.

The patient underwent oncologic consultation due to the complex history and clinical manifestations. The hepatic lesion was highly suspicious for malignancy and differential diagnosis pointed to breast cancer metastasis as the most probable scenario, although HCC could not be ruled out. The calcified pancreatic lesion had rather benign features and rarity of pancreatic metastases was also evaluated. Both lesions were considered resectable according to CT findings. Minimal invasive methods of biopsies were proposed to the patient to get a definitive diagnosis. False diagnosis of hepatic metastasis from breast cancer could lead to inappropriate medical treatment with endocrine therapy and chemotherapy and would delay the appropriate treatment of primary HCC which is principally R0 surgical resection in patients with well-maintained liver function. ${ }^{17,18,20}$

Our patient denied CT-guided or laparoscopic biopsies of the lesions and since R0 surgical excision of a solitary hepatic metastasis from breast cancer is also safe and potentially beneficial, we proposed and the patient accepted to undergo surgical rapid biopsies and surgical excision of the hepatic and if needed of the pancreatic lesion during one operation. The pancreatic lesion was left intact since malignancy was not confirmed. The hepatic mass was proven to be a primary HCC. Intraoperative hepatic ultrasound confirmed that the right hepatic vein and major intrahepatic ducts were not infiltrated by the HCC. Thus we planned to perform a wide right hepatic segmentectomy (VI-VII) with a $2 \mathrm{~cm}$ margin of normal liver parenchyma which is oncologically acceptable and preserves hepatic parenchyma minimizing the risk for postoperative acute liver failure. For that purpose we used a microwave tissue coagulator which is a safe and effective method to perform ablation and transection of hepatic parenchyma in less time and with minimal heat-sink effect and intraoperative blood loss. We also combined the use of harmonic scalpel to achieve a faster and safer hepatic transection. ${ }^{21-25}$

The patient tolerated the operation well and had an uncomplicated postoperative course. Since pathology report confirmed the wide margin-free excision of the HCC and staging of the patient excluded extrahepatic disease, the oncology council denied any further adjuvant therapy at present and suggested transarterial chemoembolization or treatment with sorafenib in case of future recurrence. Two years after the operation, the patient remains disease free from both treated malignancies and under close medical follow-up. ${ }^{17,18,26,27}$

\section{Conclusion}

Patients treated for breast cancer are at a high risk for developing a second primary malignancy and they should undergo close medical follow-up which should be continued even if 5 -year survival is achieved. Differential diagnosis of hepatic lesions detected in these patients apart from liver metastases should include primary hepatic malignancies. Thus, all hepatic lesions in breast cancer patients should be evaluated with open mind, and liver biopsy should be performed to get a definitive diagnosis and implement the proper treatment strategy.

\section{Disclosure}

The authors report no conflicts of interest in this work.

\section{References}

1. Chen QW, Li HJ, Chen YN, et al. Hepatic Lesions Detected after Mastectomy, in Breast Cancer Patients with Hepatitis Background May Need to Undergo Liver Biopsy to Rule Out Second Primary Hepatocellular Carcinoma. PLoS One. 2016;11(1): $\mathrm{e} 0139782$.

2. Dong C, Chen L. Second malignancies after breast cancer: The impact of adjuvant therapy. Mol Clin Oncol. 2014;2(3):331-336.

3. Kirova YM, De Rycke Y, Gambotti L, et al; Institut Curie Breast Cancer Study Group Second malignancies after breast cancer: the impact of different treatment modalities. Br J Cancer. 2008;98(5):870-874. 
4. Margonis GA, Buettner S, Sasaki K, et al. The role of liver-directed surgery in patients with hepatic metastasis from primary breast cancer: a multi-institutional analysis. HPB (Oxford). 2016;18(8):700-705.

5. Jin X, Mu P. Targeting Breast Cancer Metastasis. Breast Cancer (Auckl). 2015;9(Suppl 1):23-34.

6. Ma R, Feng Y, Lin S, et al. Mechanisms involved in breast cancer liver metastasis. J Transl Med. 2015;13:64.

7. Noguchi M, Naruki Y, Matsuo K, et al. [A case report of triple malignant tumor including early gastric carcinoma--multiple primary malignant tumors, a survey of the literatures and a statistical study (author's trans1)]. Nihon Shokakibyo Gakkai Zasshi. 1978;75(1):71-79. Japanese.

8. Mariotto AB, Rowland JH, Ries LA, Scoppa S, Feuer EJ. Multiple cancer prevalence: a growing challenge in long-term survivorship. Cancer Epidemiol Biomarkers Prev. 2007;16(3):566-571.

9. Wyld L, Gutteridge E, Pinder SE, et al. Prognostic factors for patients with hepatic metastases from breast cancer. $\mathrm{Br} \mathrm{J}$ Cancer. 2003;89(2):284-290.

10. Eichbaum MH, Kaltwasser M, Bruckner T, de Rossi TM, Schneeweiss A, Sohn C. Prognostic factors for patients with liver metastases from breast cancer. Breast Cancer Res Treat. 2006;96(1):53-62.

11. Cassera MA, Hammill CW, Ujiki MB, Wolf RF, Swanström LL, Hansen PD. Surgical management of breast cancer liver metastases. $H P B$ (Oxford). 2011;13(4):272-278.

12. Podnos YD, Wagman LD. Surgical management of hepatic breast cancer metastases. Oncology (Williston Park). 2005;19(12):1583-1587; 1587-1588 discussion; 1590 passim.

13. Scully OJ, Bay BH, Yip G, Yu Y. Breast cancer metastasis. Cancer Genomics Proteomics. 2012;9(5):311-320.

14. Bonapasta SA, Gregori M, Lanza R, et al. Metastasis to the Pancreas from Breast Cancer: Difficulties in Diagnosis and Controversies in Treatment. Breast Care (Basel). 2010;5(3):170-173.
15. Boussios S, Zerdes I, Batsi O, et al. Pancreatic resection for renal cell carcinoma metastasis: An exceptionally rare coexistence. Int J Surg Case Rep. 2016;27:198-201.

16. Chen C, Wang X, Xiong X, et al. Targeting type I phosphatidylinositol phosphate kinase inhibits breast cancer metastasis. Oncogene. 2015;34(35):4635-4646.

17. Waller LP, Deshpande V, Pyrsopoulos N. Hepatocellular carcinoma: A comprehensive review. World J Hepatol. 2015;7(26):2648-2663.

18. Balogh J, Victor D. 3rd, Asham EH, et al. Hepatocellular carcinoma: a review. J Hepatocell Carcinoma. 2016;3:41-53.

19. Sadot E, Lee SY, Sofocleous CT, et al. Hepatic Resection or Ablation for Isolated Breast Cancer Liver Metastasis: A Case-control Study With Comparison to Medically Treated Patients. Ann Surg. 2016;264(1):147-154.

20. Lee H, Yoon JH, Kim H, et al. False Positive Diagnosis of Hepatocellular Carcinoma in Liver Resection Patients. J Korean Med Sci. 2017;32(2):315-320.

21. Tan K, du X, Yin J, et al. Microwave tissue coagulation technique in anatomical liver resection. Biomed Rep. 2014;2(2):177-182.

22. Lubner MG, Brace CL, Ziemlewicz TJ, Hinshaw JL, Lee FT. Microwave ablation of hepatic malignancy. Semin Intervent Radiol. 2013;30(1):56-66.

23. Poggi G, Tosoratti N, Montagna B, Picchi C. Microwave ablation of hepatocellular carcinoma. World J Hepatol. 2015;7(25):2578-2589.

24. Tabuse K. Basic knowledge of a microwave tissue coagulator and its clinical applications. J Hepatobiliary Pancreat Surg. 1998;5(2):165-172.

25. Poon RT. Current techniques of liver transection. HPB (Oxford). 2007;9(3): 166-173.

26. Lencioni R, Petruzzi P, Crocetti L. Chemoembolization of hepatocellular carcinoma. Semin Intervent Radiol. 2013;30(1):3-11.

27. Gholam P. The Role of Sorafenib in Hepatocellular Carcinoma. Gastroenterol Hepatol. 2015;11(4):253-255.
International Journal of General Medicine

\section{Publish your work in this journal}

The International Journal of General Medicine is an international, peer-reviewed open-access journal that focuses on general and internal medicine, pathogenesis, epidemiology, diagnosis, monitoring and treatment protocols. The journal is characterized by the rapid reporting of reviews, original research and clinical studies across all disease areas.

\section{Dovepress}

The manuscript management system is completely online and includes a very quick and fair peer-review system, which is all easy to use. Visit http://www.dovepress.com/testimonials.php to read real quotes from published authors.

Submit your manuscript here: https://www.dovepress.com/international-journal-of-general-medicine-journal 